\title{
DNA damage-induced cell death: lessons from the central nervous system
}

\author{
Helena Lobo Borges ${ }^{1,2}$, Rafael Linden ${ }^{3}$, Jean YJ Wang ${ }^{1}$ \\ ${ }^{1}$ Division of Hematology/Oncology, Moores Cancer Center, Department of Medicine, University of California, San Diego, 3855 Health \\ Sciences, La Jolla, CA 92093-0820, USA; 'Departamento de Anatomia, Instituto de Ciências Biomédicas, Universidade Federal \\ do Rio de Janeiro, Centro de Ciências da Saúde, Cidade Universitária, 21940-590 Rio de Janeiro, Brazil; ${ }^{3}$ Instituto de Biofísica \\ Carlos Chagas Filho, Universidade Federal do Rio de Janeiro, Centro de Ciências da Saúde, Cidade Universitária, 21940-590 Rio \\ de Janeiro, Brazil
}

DNA damage can, but does not always, induce cell death. While several pathways linking DNA damage signals to mitochondria-dependent and -independent death machineries have been elucidated, the connectivity of these pathways is subject to regulation by multiple other factors that are not well understood. We have proposed two conceptual models to explain the delayed and variable cell death response to DNA damage: integrative surveillance versus autonomous pathways. In this review, we discuss how these two models may explain the in vivo regulation of cell death induced by ionizing radiation (IR) in the developing central nervous system, where the death response is regulated by radiation dose, cell cycle status and neuronal development.

Keywords: apoptosis, ATM, ionizing radiation, neonatal retina, neuroblasts, p53, phosphorylation Cell Research (2008) 18:17-26. doi: 10.1038/cr.2007.110; published online 18 December 2007

\section{Introduction}

The integrity of the genome is fundamental to the propagation of life, illustrated by the complexity of the DNA replication and repair mechanisms discussed in other articles of this volume. Besides the basal levels of DNA lesions generated from normal cell metabolism, damage is further induced by genotoxic stress. Failure to repair damaged DNA gives rise to mutations and chromosomal abnormalities. An important biological strategy to guard against damaged and mutated cells is the activation of programmed cell death in response to DNA damage [1-3]. Furthermore, genotoxic agents such as ionizing radiation (IR) and chemotherapeutic drugs are the mainstay of cancer therapy because they can trigger programmed cell death $[4,5]$. Defects in DNA damage-induced cell death, therefore, not only increase the risk of cancer but also reduce the efficacy of cancer therapy. Thus, understanding how DNA damage causes cell death has important translational potential in human diseases such as cancer.

The cell death response to DNA damage is only one of

Correspondence: Jean YJ Wang

E-mail: jywang@ucsd.edu several biological outcomes that can result from cellular exposure to genotoxic stress. These biological responses are temporally regulated such that DNA repair and cell cycle arrest are activated immediately following DNA damage [6]. Successful execution of these immediate early pathways protects the damaged cell, allowing for recovery and resumption of cell proliferation (Figure 1). Generally speaking, cell death is a delayed response to DNA damage, resulting in elimination of the damaged cell (Figure 1). The cell-protective and cell-destructive responses to DNA damage are downstream of a common signal transduction network that has been studied intensively in recent years (Figure 1) (reviewed in Huen and Chen, in this issue) [2, 3, 7-17]. While most protein components of this signaling network have been identified, the current knowledge does not explain when and why a damaged cell will choose to die. In other words, we have not yet elucidated the rules that govern the cell death response to DNA damage.

\section{Two models for how death response to DNA damage is regulated}

We have previously proposed two conceptual models for how DNA repair, cell cycle arrest and death responses to DNA damage may be coordinated [6]. The first model, 

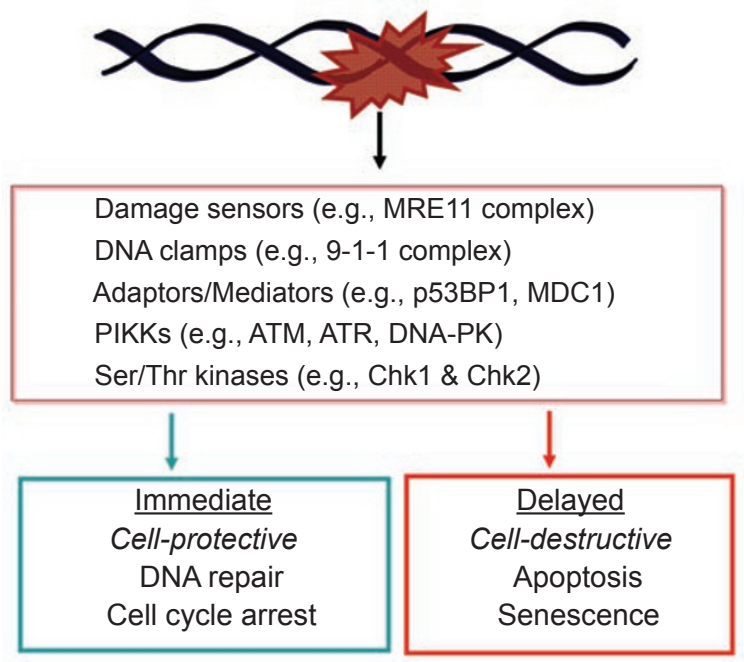

Figure 1 DNA damage activates opposing biological outcomes through a common signaling network. Components of the DNA damage signal transduction network are listed in the box. Examples of proteins that sense DNA lesions, e.g., the Mre11 complex that detects strand breaks, and that assemble protein complexes, e.g., the 9-1-1 complex, p53BP1 and MDC1 are provided. The sensors, clamps and adaptors/mediators facilitate the activation of apical kinases belonging to the PIKK family, including ATM, ATR and DNA-PK. The PIKKs phosphorylate a large number of substrate proteins; among them are Chk1 and Chk2, which are serine/threonine kinases. The activation of DNA repair and cell cycle arrest is an immediate response to DNA damage, and these responses are designed to protect a damaged cell and to promote its recovery. The activation of apoptosis or senescence occurs later in time. These delayed responses are designed to eliminate a damaged cell. It is interesting to note that the DNA damage-signaling network can lead to two opposite biological outcomes.

which we termed "integrative surveillance", proposes that the DNA damage signaling network functions as a regulatory hub, which continually monitors DNA integrity to control the choice between cell cycle arrest and cell death (Figure 2A). This concept underlies the statement "cells die when the damage is irreparable", which is often found in research papers on cellular responses to DNA damage. Although this intuitive concept of integrative surveillance is widely accepted, there is currently no direct evidence of an irreparable DNA lesion, nor is there any biochemical mechanism for how the DNA damage signaling network distinguishes between "reparable" versus "irreparable" DNA lesions. Nevertheless, integrative surveillance has remained a plausible, and seldom challenged, explanation for when and how a damaged cell may choose to commit suicide.

We have proposed an alternative concept, which we termed "autonomous pathways" [6], which departs from the intuitive idea regarding the death response to DNA damage (Figure 2B). This model does not invoke a biochemical mechanism to detect "irreparable" DNA lesions. Instead, it is proposed that the death-signaling pathways are designed with intrinsic delay mechanisms (Figure 2B). In the autonomous pathways model, the DNA damagesignaling network activates DNA repair and cell death simultaneously and automatically, which is consistent with the current evidence [2, 3, 7-17]. The delayed onset of cell death can be due to (a) a requirement for new gene expression; because accumulation of RNA transcripts and protein products naturally takes time, the death response is delayed; (b) built-in feedback loops in the death response pathways such that death only occurs when the negative
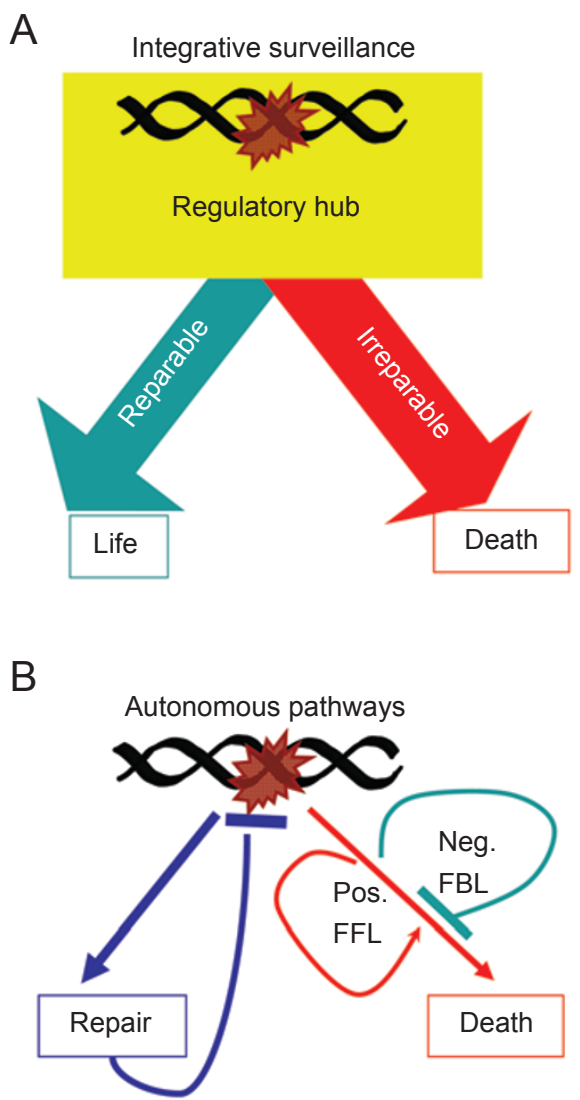

Figure 2 Conceptual models for the coordination of DNA repair and cell death. (A) The "integrative surveillance" model: the DNA damage signaling network functions as a regulatory hub that makes calculated decisions, based on the status of DNA repair and the nature of DNA lesions to choose between life or death. (B) The "autonomous pathways" model: DNA damage activates DNA repair and cell death independently and automatically. The repair pathway extinguishes the damage signal. The death pathways (illustrated as a single entity to clarify the concept) contain intrinsic negative feedback loops (Neg. FBL) and positive feed-forward loops (Pos. FFL). These positive and negative regulatory loops are the causes of the delayed death response to DNA damage. 
feedback is overridden; and (c) a requirement for positive feed-forward loops to raise the death signals above a threshold that can overcome existing survival signals. In this model, the continuous and repeated activation of the death signaling pathways, resulting from the continuous presence of DNA lesions, is necessary to either stimulate the positive feed-forward or dampen the negative feedback loops, and thus death is a delayed response that correlates with the persistence of DNA damage.

In this review, we will examine these two conceptual models for their capacity to explain DNA damage-induced cell death in the developing central nervous system (CNS) of rodents. We chose to limit the discussion to in vivo results because the literature on DNA damage-induced death in cultured cells is too vast and variable. Before discussing the in vivo results, we will briefly review the current knowledge on the death machines that DNA damage can activate in mammalian cells.

\section{DNA damage-activated death machines}

Apoptosis is a morphological descriptor of a form of cell death that involves nuclear condensation and DNA fragmentation. Apoptotic death is mainly executed through the activation of caspases, and this physiological form of cell death is employed during development to sculpture the body, a good example being the formation of digits [18]. Genotoxic stress can activate apoptotic cell death through caspase-dependent or independent pathways.

\section{Activation of caspase-dependent apoptosis by DNA dam- age}

The caspase-dependent apoptosis machine was discovered from studies of developmentally programmed cell death in the nematode Caenorhabditis elegans [19, 20]. In $C$. elegans, the evolutionarily conserved DNA damage-signaling network activates DNA repair and cell cycle checkpoints, but it does not activate caspase-dependent apoptosis in somatic cells. In this organism, genotoxins activate caspase-dependent apoptosis only in the germ cells [21-23]. Therefore, the DNA damage response pathways of $C$. elegans provide strong evidence for the statement that DNA damage does not always induce programmed cell death.

In the C. elegans germ cells and in mammalian cells, DNA damage activates caspases through p53. The p53 protein is a downstream target of the DNA damage signaling network, which causes the stabilization and activation of the transcriptional function of p53 to stimulate the expression of pro-apoptotic genes, notably Puma $[3,24]$ and Noxa [3]. Puma and Noxa encode BH3-only proteins that inactivate anti-apoptotic Bcl2-family proteins and ac- tivate $\mathrm{Bax}$ or Bak to cause cytochrome $\mathrm{C}$ release from the mitochondria [25]. Cytochrome $\mathrm{C}$ binds to and stimulates Apaf-1 to assemble the apoptosome, leading to the activation of caspases [26] (Figure 3). In addition to p53, the mammalian genome encodes two other related transcription factors, $\mathrm{p} 63$ and p73, which appear to also stimulate pro-apoptotic gene expression. Fibroblasts derived from p63/p73 double knockout mouse embryos (MEF) exhibit apoptosis defects that are similar to p53 knockout MEFs $[27,28]$. The interplay among the three members of the p53 family in regulating the expression of pro-apoptotic genes is still under investigation. Clearly, p53 is necessary for DNA damage to activate caspase-dependent apoptosis; however, physiological levels of p53 may not be sufficient to induce cell death.

\section{Negative and positive loops activated by p53}

With regard to the model that death signaling is regulated by negative and positive feedback loops (Figure 2B), it is interesting to note that the $\mathrm{p} 53-\mathrm{Mdm} 2$ negative feedback loop is activated in response to DNA damage [29,30]. This p53-Mdm2 negative feedback loop can create waves of p53 activation following DNA damage [31]. Recently, it has been discovered that DNA damage activates p53-dependent expression of specific microRNAs, which have pro-apoptotic functions $[32,33]$. These p53-induced microRNAs may amplify the pro-apoptotic signals by targeting a network of existing transcripts in response to DNA damage, and thus supports the notion of a positive feed-forward loop in p53-mediated apoptotic pathways [32, 33].

\section{Activation of caspase-independent apoptosis}

DNA condensation and fragmentation can also occur through a caspase-independent mechanism (Figure 3).

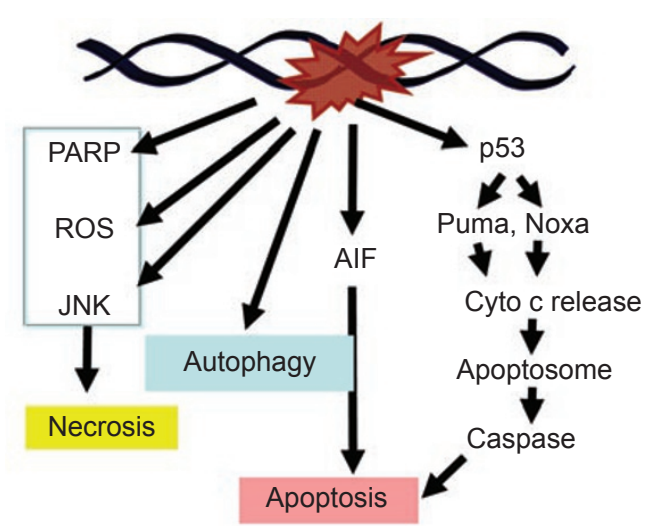

Figure 3 DNA damage-activated death machines. Summary of discussion in text on the various death mechanisms that have been shown to be activated by DNA damage in mammalian cell lines. 
The best-studied pathway involves the nuclear translocation of apoptosis-inducing factor (AIF), a mitochondrial protein that is conserved in eukaryotic cells from yeasts to humans [34]. Nuclear translocation of AIF in response to genotoxins and other types of stress signals occurs in yeast and mammalian cells $[34,35]$. Because the yeast genome encodes AIF but not caspases or Bcl2-family members, the AIF-mediated apoptosis-like cell death appears to be a more ancient death mechanism that can be triggered by genotoxins. Nuclear translocation of AIF is associated with nuclear condensation and DNA fragmentation [35], which are commonly used as indicators of apoptotic cell death [36]. Therefore, DNA damage-induced DNA fragmentation can result from the activation of caspases or the nuclear translocation of AIF.

Autophagy is a lysosomal-dependent degradative pathway that affords cell protective function under starvation or stress. However, autophagy can also cause cell death when it becomes over-activated. Autophagic cell death is characterized by the appearance of large autophagic vacuoles, i.e., the autophagosome, which is derived from part of the endoplasmic reticulum. The autophagosome fuses with the lysosome, after which its contents are degraded by lysosomal hydrolytic proteases [37]. The nucleus of a cell undergoing autophagic cell death may become pyknotic, either in early or in late stages of the degenerative process. Nevertheless, this nuclear condensation is not as common as that of apoptosis [38]. There have been several reports of induction of autophagy by treatment with anticancer drugs and/or irradiation [39]. Moreover, intensive irradiation led to cell death in MCF-7 cells accompanied by the formation of acidic vesicular organelles, whereas, following low-dose irradiation, the presence of acidic vesicular organelles correlated with an increased chance of survival, suggesting that moderate signs of autophagy may be associated with a defensive reaction of non-lethally damaged cells [38].

Autophagic cell death may be triggered by blockade of apoptosis. Indeed, caspase inhibition by zVADfmk in L929 cells resulted in autophagy-dependent cell death [40]. In addition, when etoposide-induced apoptosis was inhibited in mouse embryonic fibroblasts by deficiency of pro-apoptotic genes Bax and Bak, cell death was correlated with autophagic morphology [37, 38, 41].

Necrosis is a morphological descriptor of cell death due to loss of membrane integrity. Instead of condensation and fragmentation, necrotic cells spill their content and trigger inflammation. Necrosis can be accidental as well as programmed [42]. The production of reactive oxygen species (ROS), the sustained activation of Jun N-terminal kinase (JNK) and the activation of poly(ADP-ribose) polymerase1 (PARP-1) have each been shown to cause necrotic cell death in response to DNA damage [42-44] (Figure 3).
PARP-1 is an abundant nuclear protein that is activated by single-stranded breaks; excessive PARP-1 activity is thought to activate necrosis by depleting NAD $[45,46]$. The pathways linking DNA damage to ROS, JNK and PARP have been extensively studied; however, the biochemical mechanisms that ultimately and actively induce necrotic cell death remain to be elucidated.

Mitotic death has been widely used in the scientific literature to describe clonogenic failure caused by DNA damage. Unfortunately, mitotic death inferred from clonogenic survival assays does not distinguish between (a) the actual death of damaged cells resulting from programmed apoptosis/necrosis and (b) irreversible growth arrest that does not involve cell death [16]. Mitotic death may also describe mitotic catastrophe, which is a consequence of defects in $\mathrm{G} 2 / \mathrm{M}$ and spindle checkpoint responses [10, $13,14]$.

In this review, we will limit the discussion to in vivo results where DNA damage-induced death is measured by nuclear condensation (pycnotic nuclei) and DNA fragmentation (TUNEL assay). These methods mostly measure caspase-dependent apoptosis, but they can also report caspase-independent apoptosis and programmed necrosis. We will also limit the discussion to the death response induced by IR because this physical agent penetrates the organisms without the complications of cellular transport systems. The discussion is focused on IR-induced cell death in genetically engineered mouse strains, which provide genetic information to test our models.

\section{In vivo cell death response to $I R$}

IR has widely been used as an effective method to induce DNA damage in vivo, since all cells are equally exposed, regardless of external factors such as drug uptake, detoxification or metabolism. Several organs have been employed as in vivo models of IR-induced apoptosis: the gastro-intestinal (GI) tract, the thymus, the developing liver and the developing CNS [47-50]. Among those, the developing CNS has so far been the most investigated, and thus the focus of this discussion. Although apoptosis is a cellular outcome, it is influenced by the tissue context; this includes the interaction of cells with both the extracellular matrix and other cells within the complex environment of the CNS, as well as the dynamic remodeling of nervous tissue during embryonic development [51, 52]. For instance, the developing CNS is highly susceptible to DNA damage-induced apoptosis, but this sensitivity is lost after neural differentiation [53-56]. Moreover, in the adult CNS, high sensitivity to IR is primarily seen in progenitor cells located at neurogenic compartments, which might be associated with the radiation-induced cognitive impairment seen in patients after cancer treatment $[57,58]$. Therefore, 


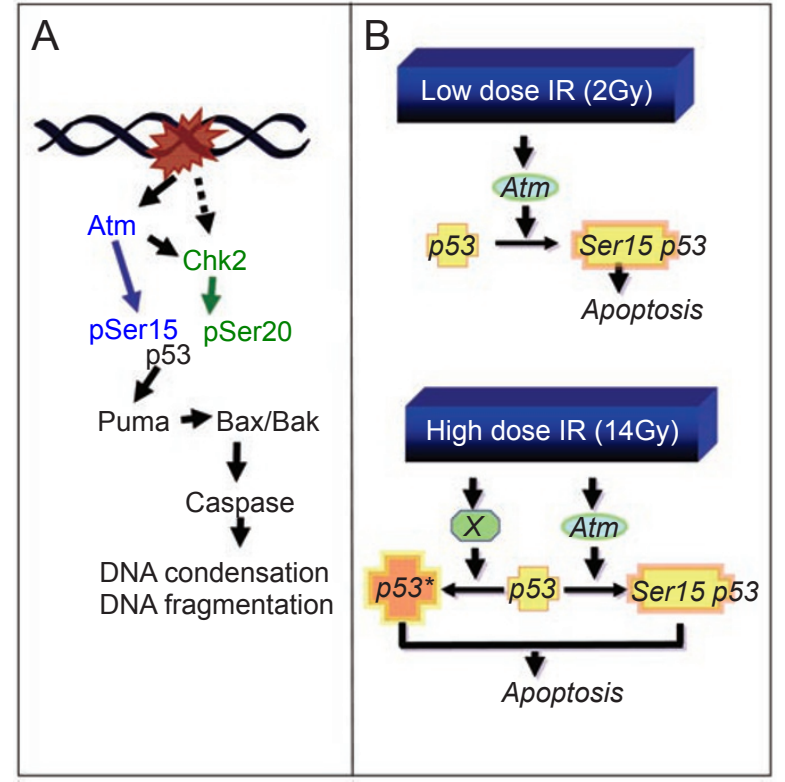

the developing CNS is a good paradigm to investigate IRinduced cell death, allowing the study of cellular responses within an intact supracellular environment. Multiple studies have addressed the genetic requirements for in vivo IR-induced apoptosis in the developing CNS, which we summarize below.

Several genes in the ATM-p53 pathway have been implicated in IR-induced apoptosis using genetically modified mice. Their contribution differed depending on the tissue involved and the IR dose used, indicating that this response is differentially and tightly regulated. For instance, while the lack of $p 53$ resulted in complete resistance to different doses of IR ( 2 and 14 Gy), Atm deficiency conferred only partial resistance, suggesting that other pathways converge upon p53 to induce cell death (Figures 3 and 4) [48, 50]. Puma-l- mice showed a remarkable defect in cell death in all areas of the developing CNS examined after 14 Gy of IR (Figures 3 and 4A). This defect was comparable to that of $p 53-/-$ mice, suggesting that $\mathrm{p} 53$-dependent cell death is mainly mediated through activation of Puma [59] (Figure 4A). In contrast, deletion of Bax, another p53-activated pro-apoptotic protein, led to a partial defect in IR-induced apoptosis in the developing cerebellum, but not in the immature retina [60] (Figure 4A). Moreover, mice nullizygous for both Bax and Atm showed a higher reduction of IR-induced apoptosis in the developing cerebellum than the individual knockouts [60], suggesting that Bax may also be activated in an ATM-independent manner (Figure 4A). Deficiency in both Bax and in another pro-apoptotic protein from the same family, Bak, caused resistance to cell death
Figure 4 Role of the ATM-p53 pathway in IR-induced apoptosis of developing CNS. The phosphorylation sites are shown for human p53. (A) Summary of genetic studies that have identified players in IR-induced apoptosis in the developing CNS under variable doses (2, 5, 8, 10 and $14 \mathrm{~Gy}$, see text for details). (B) Radiation dose affects the requirements for Atm and p53 in apoptosis. At 2 Gy, loss of Atm or p53, or serine-18 phosphorylation site in p53 (corresponding to human p53 serine-15 phosphorylation site), is sufficient to inactivate the apoptotic response to IR. While Atm through Chk2 can lead to p53 phosphorylation at Ser-23 (corresponding to human p53 serine-20), mutation at this site does not affect the apoptotic response in the developing retinas. With 2 Gy of IR, p53-heterozygosity is already showing defect in IR-induced apoptosis. Thus, IR-induced activation of ATM kinase and phosphorylation at serine-18 of a full complement of p53 protein from two wild-type alleles is required to induce apoptosis. At $14 \mathrm{~Gy}$, Atm contributes to but is not required for apoptosis, whereas p53 is still required and p53 heterozygosity is sufficient to cause apoptosis. It thus appears that high-dose IR can super-activate p53 through Atm-dependent and independent (X) mechanisms such that half of the amount of p53 is sufficient to cause apoptosis.

under several conditions, such as IR-induced apoptosis, cell death induced by BH3-only members, and developmental cell death in the CNS and other areas, albeit the single mutations conferred limited apoptotic defects [61-64]. This indicates that Bax and Bak have redundant functions in distinct death-inducing pathways (Figure 4A).

ATM can contribute to $\mathrm{p} 53$ activation both directly and indirectly, by activation of Chk 2 kinase. Knockout mice for Chk2 were defective in apoptosis induced by 5-8 Gy of IR in all areas of the developing CNS examined [65]. Thus, similar to p53, Chk2 is required for IR-induced apoptosis (Figure 4A). Chk2 directly phosphorylates p 53 at serine-23 (Ser-20 in humans), but mutation of p53 at this site has little effect on cell death in embryonic cells and thymocytes [66]. The effect of Ser-23 phosphorylation is, however, synergistic with phosphorylation at serine-18 (Ser-15 in human p53), since knock-in mutations in both sites completely rescue the embryonic lethality of Xrcc4-/- mice, which die of massive p53-dependent apoptosis as a result of DNA repair defects [49]. Therefore, the contribution of Chk2 to the induction of apoptosis may either be cell type specific or involve phosphorylation of $\mathrm{p} 53$ at sites other than serine18 and Ser-23 [67, 68]. No studies have directly compared CNS responses to IR in Atm-/- and Chk2-/- mice, or the phenotype of combined Atm-/-Chk2-/- mice after IR. Thus, it is not clear whether the contribution of Chk2 to this process in CNS is dependent on ATM only, or whether another pathway is involved.

Signal transduction through $\mathrm{p} 53$ is achieved by posttranslational modifications such as those at the phosphory- 
lation sites mentioned above (see also Huen and Chen, in this volume). The importance of several post-translational modifications in p53 was investigated in vivo by taking advantage of knock-in mutations; these analyses aimed at the relevance of both phosphorylation as well as lysine acetylation for p53 functions. For instance, Lys320 of human $\mathrm{p} 53$ is acetylated in vitro by PCAF, the p300/CBPassociated factor, and this site is also acetylated in vivo following DNA damage [69]. The corresponding lysine residue of mouse p53, Lys317, is also acetylated in vivo after DNA damage [70]. Analysis of the p53-dependent responses to DNA damage in mice containing a knock-in p53 mutation at this site (Lys317Arg) indicated that this acetylation negatively regulates $\mathrm{p} 53$-induced apoptosis in both the GI tract and developing retina [71]. Therefore, post-translational modifications of p53 can be used both positively and negatively to regulate apoptosis. These findings further illustrate the complex regulation of the DNA damage response.

As described above, p53 is a central component of IRinduced apoptosis. Nonetheless, other members of the p53 family also play an important role in this process. Combined deficiency of both p63 and p73 also conferred complete resistance to apoptosis following 5 Gy of IR in the embryonic cerebral cortex [27], whereas defects in either $p 63$ or $p 73$ led only to partial resistance. These results do not diminish the importance of $\mathrm{p} 53$ in IR-induced cell death, but suggest that $\mathrm{p} 63$ and $\mathrm{p} 73$ contribute to $\mathrm{p} 53$-mediated cell death [27]. Some genes, such as Noxa and Bax, failed to be efficiently upregulated in the absence of p63 and p73, whereas other genes, such as p21 and Mdm2, were expressed at wild-type levels following IR. These data suggest that the p53 family works in cooperation to induce cell death.

Studies of IR-induced apoptosis in vivo have been performed with differing doses of radiation (2-14 Gy), and contrasting results were found depending on the dose used. For instance, following 2 or 14 Gy IR, lack of Atm conferred partial resistance to cell death. However, following 14 Gy of IR, Atm was necessary for apoptosis in areas of the CNS containing migrating and differentiating cells [72], whereas, following 2 Gy of IR, Atm was also necessary for apoptosis in proliferating areas (see below) [48]. Another example of requirements of dose-dependent apoptosis was observed in $p 53$ heterozygous retinas. After 2 Gy of IR, $p 53+/-$ and $p 53-/-$ retinas exhibited a similar degree of resistance to apoptosis, suggesting that the full complement of $\mathrm{p} 53$ protein level is required for low-dose radiation to induce apoptosis. After 14 Gy of IR, p53+/- retina underwent rates of apoptosis similar to $p 53+/+$ retinas, whereas $p 53-/-$ retinas remained resistant to apoptosis. Therefore, a single allele of wild-type p53 is sufficient for high-dose IR to induce apoptosis.
Another example in which IR dose influenced the requirements for cell death was found with mice mutated in p53 at serine- 18 (serine- 15 in human p53), which is a direct target of phosphorylation by ATM [70] (Figure 4A). Homozygous Ser18Ala knock-in mutant mice were strongly defective in 2-Gy-induced apoptosis, but showed nearly wild-type sensitivity following 14 Gy of IR. Therefore, phosphorylation of p53 at serine-18 is required for lowdose IR-induced neuronal cell death, but it is dispensable following 14 Gy IR. These data suggest that high-dose IR may activate $\mathrm{p} 53$ through mechanisms other than serine-18 phosphorylation to cause apoptosis (Figure 4B).

These genetic studies clearly demonstrate that the apoptotic response to DNA damage within the developing CNS requires upstream kinases such as ATM and Chk2, the p53 protein and its related $\mathrm{p} 63$ and $\mathrm{p} 73$, and downstream transcription targets such as Puma. The fact that the apoptotic response requires the same upstream kinases that are also involved in DNA repair and checkpoint responses suggests that the pathways to apoptosis are triggered at the same time as that of repair and cell cycle arrest.

\section{Regulation of IR-induced apoptosis in the develop- ing retina}

The developing retina of neonatal rats and mice exhibits a complex death response to IR. Apoptosis is triggered in the neonatal rodent retina at IR doses as low as $0.5-2$ Gy [55]. In newborn rats and mice, the neonatal retina is composed of three layers of cells: a ganglion cell layer (GCL), an incipient inner nuclear layer (INL) and a neuroblastic layer (NBL) (Figure 5A). Post-mitotic cells in an advanced stage of differentiation reside in the GCL and INL, whereas the NBL contains both proliferating neuroblasts and undifferentiated post-mitotic cells $[55,73,74]$. Therefore, the layers of the developing retina correspond to distinct stages of cell differentiation (Figure 5A). The neonatal retina exhibits yet another characteristic that imparts useful information regarding the cell cycle. The proliferating neuroblasts have elongated profiles, and their nuclei undergo intracellular migration according to the cell cycle phases. The nuclei that are undergoing DNA synthesis are found in the inner region of the NBL, whereas those undergoing mitosis are exclusively located to the outermost margin of the NBL. Nuclei in the G1- and G2-phases migrate towards the inner and outer margins of the NBL, respectively (Figure 5A). This ballet of nuclei is known as interkinetic nuclear migration [75].

We have characterized the retinal apoptotic response to IR in newborn rats and mice $[48,55]$. Both species exhibited similar spatial and temporal responses, which were influenced by developmental stage as well as by the 
A

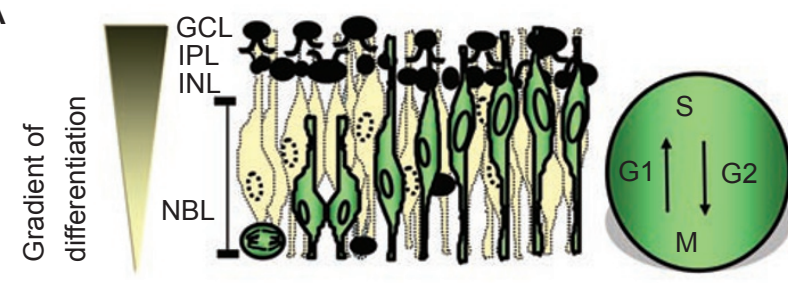

B

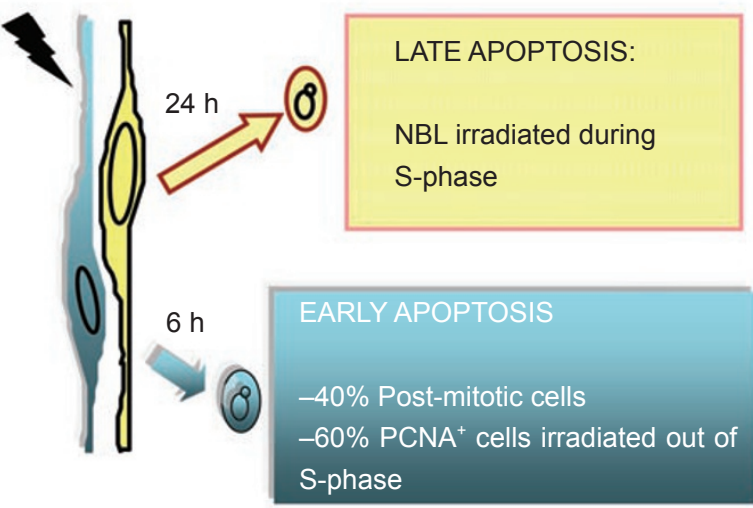

Figure $\mathbf{5}$ Two waves of IR-induced apoptosis in the developing retina. (A) Cell layers in the developing retina of newborn mice or rats, and the interkinetic nuclear migration (cell cycle-related nuclear migration). The GCL and INL contain cells in an advanced stage of maturation. The IPL, inner plexiform layer, is a region of connections among cells. The NBL contains proliferating neuroblasts and recent post-mitotic cells. The gradient of differentiation is indicated on the left. Proliferating neuroblasts (white cells) and their nuclear migration along the phases of the cell cycle (G1, S, $\mathrm{G} 2, \mathrm{M}$ ) are depicted. Black cells in the NBL are recent post-mitotic cells ( $\mathrm{G} 0$ cells) that have exited the cell cycle and begun the process of terminal differentiation. (B) Two waves of apoptosis are induced by a single dose of IR as low as $0.5 \mathrm{~Gy}$. Early apoptosis (6 h post-IR) involves recent post-mitotic cells (PCNA negative cells, also negative for differentiation markers), and proliferating neuroblasts not in S-phase at the time of irradiation (PCNA positive, BrdU negative). In this early wave, apoptosis correlates with an increase in lipid peroxidation and can be blocked by the anti-oxidant PDTC. Early apoptosis is also inhibited by blockage of protein synthesis (cycloheximide, CHX). The later wave of apoptosis (24 $\mathrm{h}$ post-IR) is comprised of neuroblasts that are in S-phase cells at the time of irradiation (PCNA positive, BrdU positive). Cells in the late wave of apoptosis are proliferating because they can be labeled with BrdU as late as $21.5 \mathrm{~h}$ post-IR. Because the late wave of apoptosis occurs in the S-phase zone, it suggests that apoptosis is triggered as cells re-enter the second S-phase after IR. However, we cannot rule out the possibility that these damaged cells remain in S-phase and continue to synthesize DNA for $24 \mathrm{~h}$ before dying to IR-induced damage. The later wave of apoptosis is not inhibited by the anti-oxidant PDTC but it is blocked by cycloheximide. Both waves of apoptosis require Atm and Serine-18 phosphorylation of p53 [48]. cell cycle phases. After 2 Gy IR, cells in the NBL displayed two separate waves of cell death characterized by internucleosomal fragmentation of DNA consistent with caspase-dependent apoptosis. By taking advantage of the well-characterized cell cycle-related migration in the NBL, it was possible to infer the cell cycle phase of cells in each apoptotic wave by the location of their nuclei. There was a gradient of cell death towards the outer edge of the NBL $6 \mathrm{~h}$ after IR, the location of non-S-phase cells, whereas the gradient of cell death observed $24 \mathrm{~h}$ after IR was directed at the inner margin of the NBL, which is the location of S phase cells. The cell cycle phase predicted by the position of dying nuclei was confirmed by pulse labeling mice with bromo-deoxy-uridine (BrdU), an analog of thymidine, $1 \mathrm{~h}$ before IR. At $6 \mathrm{~h}$ after IR early apoptotic cells were mostly BrdU-negative, whereas at $24 \mathrm{~h}$ the majority of apoptotic cells were labeled with BrdU. These waves are likely independent, since an increase in lipid peroxidation was detected at $6 \mathrm{~h}$ but not at $24 \mathrm{~h}$ after IR. Accordingly, the antioxidant pyrrolidinedithiocarbamate (PDTC) prevented the first, but not the second wave of apoptosis [55] (Figure 5B).

The studies of IR-induced cell death in the developing retina have demonstrated that the dying retinal cells could be grouped into at least two populations that exhibit spatially and temporally distinct apoptotic responses to DNA damage. The early wave of apoptosis involved recent post-mitotic cells (cells that just left the cell cycle but did not have time to express any differentiation marker) and proliferating cells not in S-phase. The second wave of apoptosis, occurring $24 \mathrm{~h}$ post-IR, involved proliferating cells, including those in S-phase at the time of irradiation $[48,55]$. Cells dying at $24 \mathrm{~h}$ post-IR appear to be actively cycling because they can be labeled with BrdU given to the whole animal at $21.5 \mathrm{~h}$ post-IR [48]. Thus, the timing of cell death following DNA damage induced by IR is dependent on both the cell cycle phase and the stage of differentiation (Figure 5A and 5B) [48, 55]. It is likely that the differential timing of cell death is due to factors other than the extent of DNA lesions, since the damage is induced by whole-body IR of the newborn animals.

The simplest interpretation of the complex death response in the developing retinal tissue is that the early and later waves of death are triggered by different mechanisms. However, genetic studies have shown that the ATM-p53 pathway is required for both waves of programmed cell death [48]. Furthermore, the two waves of cell death exhibit similar phenotypic modulation by $p 53$ gene dosage, p53-Ser18Ala mutation and Atm mutation. The fact that the heterogeneous death responses in the developing retina are triggered by the same pathway strongly support the concept that death-signaling pathway is subjected to modulation 
by other factors.

\section{Conclusion and perspectives}

The two conceptual models illustrated in Figure 2 can each explain the two waves of death response, but they make different predictions.

According to the integrative surveillance model, cells that die $24 \mathrm{~h}$ following IR, i.e., cells that are engaged in DNA synthesis when exposed to IR, may possess a higher level of repair activities and thus delay the formation of "irreparable DNA lesions". By contrast, recently post-mitotic cells or proliferating cells that are not in S-phase when IR is given may be inefficient at repairing DNA lesions and hence generate "irreparable" lesions at a much earlier time. Given the fact that cycloheximide can block IR-induced retinal cell death, and that gene expression takes a few hours to complete, the death response at $6 \mathrm{~h}$ post-IR is mostly likely triggered instantaneously following irradiation. Since the early wave of cell death can be induced by IR dose as low as $0.5 \mathrm{~Gy}$, which does not cause significant mitotic death in culture cells, the retinal cells may be uniquely crippled in DNA repair. If so, the developing retinal cells would be expected to contain a high level of irreparable lesions after 0.5 Gy of IR. Biochemical analyses of DNA lesions in the developing retina following IR may therefore shed light on the nature of "irreparable" lesions that can trigger programmed cell death. An important caveat to the argument that post-mitotic cells and non-S-phase cells cannot repair 0.5 Gy IR-induced DNA lesions is the fact that the mature post-mitotic neurons in the GCL layer of the developing retina (Figure 5A) are completely resistant to IR-induced apoptosis. Thus, acceptance of the integrative surveillance model would invoke inactivation of DNA repair in post-mitotic and non-S-phase cells and reactivation of DNA repair in terminally differentiated and S-phase cells.

According to the autonomous pathways model, the negative feedback loops that delay the execution of cell death downstream of p53 may be absent from the recently post-mitotic or non-S-phase cells. Alternatively, the survival threshold is either non-existing or set at a low level in these cells such that a one-time activation of $\mathrm{p} 53$ would be sufficient to activate cell death, through the transcriptional upregulation and accumulation of Puma alone, without the need for positive feed-forward loops. With S-phase cells, on the other hand, feedback loops and/or survival thresholds are present to inhibit death signaling from $\mathrm{p} 53$. These antiapoptotic mechanisms may decay with time, thus leading to death at $24 \mathrm{~h}$ post-IR.

While the death response is subjected to regulation by cell-intrinsic factors such as the cell cycle phase and/or the stage of differentiation, we need to keep in mind that the apoptotic response is also modulated by cell-extrinsic factors, such as the production of death ligands, for example, FAS ligand, that activate death receptors $[76,77]$, or adhesion signals generated through cell-matrix and cellcell interactions $[78,79]$. As additional regulatory factors of DNA damage-induced cell death are identified through studies with cultured cell lines, the developing retina of rodents will remain an excellent experimental system to test the in vivo validity of the in vitro results.

\section{Acknowledgments}

We thank Dr Susana Chaves (The Scripps Research Institute, USA) for critical reading of this manuscript. Helena L Borges was supported by CAPES and CNPq fellowships, Brazil. Rafael Linden is supported by CNPq, FAPERJ and PRONEX-MCT, Brazil. Jean Y Wang is supported by grants from the National Cancer Institute, USA.

\section{References}

1 Rich T, Allen RL, Wyllie AH. Defying death after DNA damage. Nature 2000; 407:777-783.

2 Blank M, Shiloh Y. Programs for cell death: apoptosis is only one way to go. Cell Cycle 2007; 6:686-695.

3 Roos WP, Kaina B. DNA damage-induced cell death by apoptosis. Trends Mol Med 2006; 12:440-450.

4 Bentle MS, Bey EA, Dong Y, Reinicke KE, Boothman DA. New tricks for old drugs: the anticarcinogenic potential of DNA repair inhibitors. J Mol Histol 2006; 37:203-218.

5 Michod D, Widmann C. DNA-damage sensitizers: potential new therapeutical tools to improve chemotherapy. Crit Rev Oncol Hematol 2007; 63:160-171.

6 Wang JY, Cho SK. Coordination of repair, checkpoint, and cell death responses to DNA damage. Adv Protein Chem 2004; 69:101-135.

7 Ben-Porath I, Weinberg RA. The signals and pathways activating cellular senescence. Int J Biochem Cell Biol 2005; 37:961-976.

8 Jeggo P, Lobrich M. Radiation-induced DNA damage responses. Radiat Prot Dosimetry 2006; 122:124-127.

9 Lavin MF, Kozlov S. ATM activation and DNA damage response. Cell Cycle 2007; 6:931-942.

10 Bartek J, Lukas J. DNA damage checkpoints: from initiation to recovery or adaptation. Curr Opin Cell Biol 2007; 19:238-245.

11 Rudolph J. Inhibiting transient protein-protein interactions: lessons from the Cdc 25 protein tyrosine phosphatases. Nat Rev Cancer 2007; 7:202-211.

12 Boutros R, Lobjois V, Ducommun B. CDC25 phosphatases in cancer cells: key players? Good targets? Nat Rev Cancer 2007; 7:495-507.

13 Harrison JC, Haber JE. Surviving the breakup: the DNA damage checkpoint. Annu Rev Genet 2006; 40:209-235.

14 Mansilla S, Bataller M, Portugal J. Mitotic catastrophe as a consequence of chemotherapy. Anticancer Agents Med Chem 2006; 6:589-602.

15 Short SC, Bourne S, Martindale C, Woodcock M, Jackson SP. DNA damage responses at low radiation doses. Radiat Res 2005; 
164:292-302.

16 Lou Z, Chen J. Cellular senescence and DNA repair. Exp Cell Res 2006; 312:2641-2646.

17 Hurley PJ, Bunz F. ATM and ATR: components of an integrated circuit. Cell Cycle 2007; 6:414-417.

18 Chen Y, Zhao X. Shaping limbs by apoptosis. J Exp Zool 1998; 282:691-702.

19 Kumar S. Caspase function in programmed cell death. Cell Death Differ 2007; 14:32-43.

20 Domingos PM, Steller H. Pathways regulating apoptosis during patterning and development. Curr Opin Genet Dev 2007; 17:294299.

21 Schumacher B, Hofmann K, Boulton S, Gartner A. The C. elegans homolog of the p53 tumor suppressor is required for DNA damage-induced apoptosis. Curr Biol 2001; 11:1722-1727.

22 Schumacher B, Schertel C, Wittenburg N, et al. C. elegans ced13 can promote apoptosis and is induced in response to DNA damage. Cell Death Differ 2005; 12:153-161.

23 Salinas LS, Maldonado E, Navarro RE. Stress-induced germ cell apoptosis by a p53 independent pathway in Caenorhabditis elegans. Cell Death Differ 2006; 13:2129-2139.

24 Yu J, Zhang L. No PUMA, no death: implications for p53-dependent apoptosis. Cancer Cell 2003; 4:248-249.

25 Abu-Qare AW, Abou-Donia MB. Biomarkers of apoptosis: release of cytochrome c, activation of caspase-3, induction of 8-hydroxy-2'-deoxyguanosine, increased 3-nitrotyrosine, and alteration of p53 gene. J Toxicol Environ Health B Crit Rev 2001; 4:313-332.

26 Hajra KM, Liu JR. Apoptosome dysfunction in human cancer. Apoptosis 2004; 9:691-704.

27 Flores ER, Tsai KY, Crowley D, et al. p63 and p73 are required for $\mathrm{p} 53$-dependent apoptosis in response to DNA damage. Nature 2002; 416:560-564.

28 Deyoung MP, Ellisen LW. p63 and p73 in human cancer: defining the network. Oncogene 2007; 26:5169-5183.

$29 \mathrm{Lu}$ X, Ma O, Nguyen TA, Jones SN, Oren M, Donehower LA. The Wip1 phosphatase acts as a gatekeeper in the p53-Mdm2 autoregulatory loop. Cancer Cell 2007; 12:342-354.

30 Aylon Y, Oren M. Living with p53, dying of p53. Cell 2007; 130:597-600.

31 Lahav G, Rosenfeld N, Sigal A, et al. Dynamics of the p53-Mdm2 feedback loop in individual cells. Nat Genet 2004; 36:147150 .

$32 \mathrm{He}$ L, He X, Lim LP, et al. A microRNA component of the p53 tumour suppressor network. Nature 2007; 447:1130-1134.

33 Raver-Shapira N, Marciano E, Meiri E, et al. Transcriptional activation of miR-34a contributes to $\mathrm{p} 53$-mediated apoptosis. Mol Cell 2007; 26:731-743.

34 Cande C, Cecconi F, Dessen P, Kroemer G. Apoptosis-inducing factor (AIF): key to the conserved caspase-independent pathways of cell death? J Cell Sci 2002; 115:4727-4734.

35 Lorenzo HK, Susin SA, Penninger J, Kroemer G. Apoptosis inducing factor (AIF): a phylogenetically old, caspase-independent effector of cell death. Cell Death Differ 1999; 6:516-524.

36 Kerr JF. History of the events leading to the formulation of the apoptosis concept. Toxicology 2002; 181-182:471-474.

37 Kim R, Emi M, Tanabe K. Caspase-dependent and -independent cell death pathways after DNA damage (Review). Oncol Rep 2005; 14:595-599.
38 Assuncao Guimaraes C, Linden R. Programmed cell deaths. Apoptosis and alternative deathstyles. Eur J Biochem 2004; 271:1638-1650.

39 Yao KC, Komata T, Kondo Y, Kanzawa T, Kondo S, Germano IM. Molecular response of human glioblastoma multiforme cells to ionizing radiation: cell cycle arrest, modulation of the expression of cyclin-dependent kinase inhibitors, and autophagy. J Neurosurg 2003; 98:378-384.

$40 \mathrm{Yu} \mathrm{L}$, Alva A, Su H, et al. Regulation of an ATG7-beclin 1 program of autophagic cell death by caspase-8. Science 2004; 304:1500-1502.

41 Bredesen DE. Key note lecture: toward a mechanistic taxonomy for cell death programs. Stroke 2007; 38:652-660.

42 Proskuryakov SY, Konoplyannikov AG, Gabai VL. Necrosis: a specific form of programmed cell death? Exp Cell Res 2003; 283:1-16.

43 Festjens N, Vanden Berghe T, Vandenabeele P. Necrosis, a wellorchestrated form of cell demise: signalling cascades, important mediators and concomitant immune response. Biochim Biophys Acta 2006; 1757:1371-1387.

44 Zong WX, Ditsworth D, Bauer DE, Wang ZQ, Thompson CB. Alkylating DNA damage stimulates a regulated form of necrotic cell death. Genes Dev 2004; 18:1272-1282.

45 Coppola S, Nosseri C, Maresca V, Ghibelli L. Different basal NAD levels determine opposite effects of poly(ADPribosyl)polymerase inhibitors on $\mathrm{H} 2 \mathrm{O} 2$-induced apoptosis. Exp Cell Res 1995; 221:462-469.

46 Watson AJ, Askew JN, Benson RS. Poly(adenosine diphosphate ribose) polymerase inhibition prevents necrosis induced by $\mathrm{H} 2 \mathrm{O} 2$ but not apoptosis. Gastroenterology 1995; 109:472-482.

47 Borges HL, Hunton IC, Wang JY. Reduction of apoptosis in Rbdeficient embryos via Abl knockout. Oncogene 2007; 26:38683877 .

48 Borges HL, Chao C, Xu Y, Linden R, Wang JY. Radiation-induced apoptosis in developing mouse retina exhibits dose-dependent requirement for ATM phosphorylation of p53. Cell Death Differ 2004; 11:494-502.

49 Chao C, Herr D, Chun J, Xu Y. Ser18 and 23 phosphorylation is required for $\mathrm{p} 53$-dependent apoptosis and tumor suppression. Embo J 2006; 25:2615-2622.

50 Herzog KH, Chong MJ, Kapsetaki M, Morgan JI, McKinnon PJ. Requirement for Atm in ionizing radiation-induced cell death in the developing central nervous system. Science 1998; 280:10891091.

51 Raff MC. Social controls on cell survival and cell death. Nature 1992; 356:397-400.

52 Linden R, Rehen SK, Chiarini LB. Apoptosis in developing retinal tissue. Prog Retin Eye Res 1999; 18:133-165.

53 Ferrer I. The effect of cycloheximide on natural and X-rayinduced cell death in the developing cerebral cortex. Brain Res 1992; 588:351-357.

54 Ferrer I, Serrano T, Rivera R, Olive M, Zujar MJ, Graus F. Radiosensitive populations and recovery in X-ray-induced apoptosis in the developing cerebellum. Acta Neuropathol 1993; 86:491500 .

55 Borges HL, Linden R. Gamma irradiation leads to two waves of apoptosis in distinct cell populations of the retina of newborn rats. J Cell Sci 1999; 112(Pt 23):4315-4324.

56 Lee Y, McKinnon PJ. Responding to DNA double strand breaks 
in the nervous system. Neuroscience 2007; 145:1365-1374.

57 Mizumatsu S, Monje ML, Morhardt DR, Rola R, Palmer TD, Fike JR. Extreme sensitivity of adult neurogenesis to low doses of X-irradiation. Cancer Res 2003; 63:4021-4027.

58 Fike JR, Rola R, Limoli CL. Radiation response of neural precursor cells. Neurosurg Clin N Am 2007; 18:115-127, x.

59 Jeffers JR, Parganas E, Lee Y, et al. Puma is an essential mediator of p53-dependent and -independent apoptotic pathways. Cancer Cell 2003; 4:321-328.

60 Chong MJ, Murray MR, Gosink EC, et al. Atm and Bax cooperate in ionizing radiation-induced apoptosis in the central nervous system. Proc Natl Acad Sci USA 2000; 97:889-894.

61 Lindsten T, Ross AJ, King A, et al. The combined functions of proapoptotic Bcl-2 family members bak and bax are essential for normal development of multiple tissues. Mol Cell 2000; 6:13891399.

62 Wei MC, Zong WX, Cheng EH, et al. Proapoptotic BAX, BAK: a requisite gateway to mitochondrial dysfunction and death. Science 2001; 292:727-730.

63 D'Sa C, Klocke BJ, Cecconi F, et al. Caspase regulation of genotoxin-induced neural precursor cell death. J Neurosci Res 2003; 74:435-445.

64 Hahn P, Lindsten T, Lyubarsky A, et al. Deficiency of Bax and Bak protects photoreceptors from light damage in vivo. Cell Death Differ 2004; 11:1192-1197.

65 Takai H, Naka K, Okada Y, et al. Chk2-deficient mice exhibit radioresistance and defective p53-mediated transcription. Embo J 2002; 21:5195-5205.

$66 \mathrm{Wu}$ Z, Earle J, Saito S, Anderson CW, Appella E, Xu Y. Mutation of mouse p53 Ser23 and the response to DNA damage. Mol Cell Biol 2002; 22:2441-2449.

67 Shieh SY, Ahn J, Tamai K, Taya Y, Prives C. The human homologs of checkpoint kinases Chk1 and Cds1 (Chk2) phosphorylate p53 at multiple DNA damage-inducible sites. Genes Dev 2000; 14:289-300.

68 Craig A, Scott M, Burch L, Smith G, Ball K, Hupp T. Allosteric effects mediate CHK2 phosphorylation of the p53 transactivation domain. EMBO Rep 2003; 4:787-792.

69 Liu L, Scolnick DM, Trievel RC, et al. p53 sites acetylated in vitro by $\mathrm{PCAF}$ and $\mathrm{p} 300$ are acetylated in vivo in response to DNA damage. Mol Cell Biol 1999; 19:1202-1209.

70 Chao C, Saito S, Anderson CW, Appella E, Xu Y. Phosphorylation of murine $\mathrm{p} 53$ at ser-18 regulates the $\mathrm{p} 53$ responses to DNA damage. Proc Natl Acad Sci USA 2000; 97:11936-11941.

71 Chao C, Wu Z, Mazur SJ, et al. Acetylation of mouse p53 at lysine 317 negatively regulates p53 apoptotic activities after DNA damage. Mol Cell Biol 2006; 26:6859-6869.

72 Lee Y, Chong MJ, McKinnon PJ. Ataxia telangiectasia mutateddependent apoptosis after genotoxic stress in the developing nervous system is determined by cellular differentiation status. $J$ Neurosci 2001; 21:6687-6693.

73 Linden R, Rehen SK, Chiarini LB, et al. Death in a dish: controls of apoptosis within the developing retinal tissue. Rev Bras Biol 1996; 56(Su 1 Pt 1):89-103.

74 Rehen SK, Neves DD, Fragel-Madeira L, Britto LR, Linden R. Selective sensitivity of early postmitotic retinal cells to apoptosis induced by inhibition of protein synthesis. Eur J Neurosci 1999; 11:4349-4356.

75 Fujita S. Kinetics of cellular proliferation. Exp Cell Res 1962; 28:52-60.

76 Mollinedo F, Gajate C. Fas/CD95 death receptor and lipid rafts: new targets for apoptosis-directed cancer therapy. Drug Resist Updat 2006; 9:51-73.

77 Ferguson TA, Griffith TS. A vision of cell death: Fas ligand and immune privilege 10 years later. Immunol Rev 2006; 213:228238.

78 Truong T, Sun G, Doorly M, Wang JY, Schwartz MA. Modulation of DNA damage-induced apoptosis by cell adhesion is independently mediated by p53 and c-Abl. Proc Natl Acad Sci USA 2003; 100:10281-10286.

79 Bewick MA, Lafrenie RM. Adhesion dependent signalling in the tumour microenvironment: the future of drug targetting. Curr Pharm Des 2006; 12:2833-2848. 